\title{
Interdisciplinary Technical Exercises for Informatics Teacher Students
}

\author{
MAKAN Gergely, ANTAL Dóra, MINGESZ Róbert, GINGL Zoltán, KOPASZ Katalin, \\ MELLÁR János, VADAI Gergely
}

\begin{abstract}
More and more of today's devices are electronic and software operated. Since they measure the signals of the real world and act as a result of processing, informatics is getting closer to interdisciplinary fields. The technology of everyday devices, automotive industry, Industry 4.0, Internet of Things (Io'T) are based also on engineering, physics, electronics, biology and other fields besides informatics. Accordingly, the interdisciplinarity is important in education too, it is essential to teach related solutions of technology for the informatics teacher students since they will certainly need it during their teaching practice. Although the development of technology is still very rapid, the main principles of operation remain the same, so education should focus on this rather than on the teaching of a current software development environment or hardware realization. Following this idea, we have worked out several laboratory exercises for our programme of informatics teacher students. During their work they construct some circuits, write the code and practice the fundamental methods of embedded software development. We report on how they could understand and learn the most important principles during working on an educational, a commercial, an industrial and a medical field related exercise. The associated experience-based learning helps systematic understanding and development of creativity. Based on our experience, students are getting more self-confident by the use of modern technical systems, which we consider particularly important.
\end{abstract}

Keywords: technical informatics, Arduino, interdisciplinary education

\section{Introduction}

One of the most important issues of education is how to adapt to the rapid development of technology. Besides the experience and knowledge related to modern tools the students live in a rapidly changing environment, they used to get huge amount of information very quickly and accordingly their attitude can also be very different than it was earlier. It is a serious problem that pupils and students feel strange in the school environment; they do not understand why to learn several parts of the curriculum. This is getting to be a common approach in public thinking too: the school should teach what is needed in practice. It is a frequent debate if it is really needed to teach more universal knowledge today rather than teaching today's solutions and the use of tools directly. Is it necessary to do elementary math calculations without any tools, to know and to be able to prove the Pythagorean theorem? Is it required to teach physics, biology, and other science subjects as a separate subject?

In the field of informatics, the problem is particularly evident: we do not know what kind of tools, programming environments will be used at an IT company a few years later. So, it seems to be the best for students to acquire such knowledge that can help them to adapt to the quickly changing environment in the future. It is important to learn logical thinking and to understand the most important operating principles as they change the least. As IT solutions are getting common in more and more fields (education, industry, medicine, communication, navigation, entertainment, etc.), a certain level of interdisciplinary knowledge and openness is very useful.

Many people find it hopeless to understand the operating principles of modern devices, they are considered as "black boxes" even more than before, it is thought we can't see the details of operation. The devices often apply the latest results of technology; their operation is related to different fields of expertise. However, if we try to find the underlying principles instead of the details of the solutions, we can get a generalization that is understandable to many, the tools are often based on

Central-European Journal of New Technologies in Research, Education and Practice

Volume 1, Number 1, 2019. 
the principles that can be observed in everyday life. It is supported by the various universal building blocks based on modern technology developed especially for education $[\underline{1}, \underline{2}, \underline{3}, \underline{4}]$. They allow students to make rather serious tools by playing and enjoying the work. Therefore, it is important in teacher education to acquire in-depth knowledge and right attitude. The high-level and extensive knowledge of teachers is essential, what can't be replaced by the knowledge of modern educational methods, it can only be made more efficient by these. Our research group [ $\underline{5}$ has developed its activities according to the above. In order to support the education of informatics teacher students we develop courses, educational materials, tools and methods. We focus on practical education, and we aim to teach the required theoretical background and universal principles that can be learnt more efficiently during laboratory practices.

\section{Technical methods and tools for informatics teacher training}

The vast majority of today's devices are electronic, include processors, and are software operated. All the devices around us will be like this in the near future, regardless of size and application field. However, the construction and operation principles can be relatively simple and universal, as it is shown in Figure 1. Real-world signals can be translated to signals that can be handled by electronics and then these can be converted to numbers to allow digital signal processing. As a result, information is obtained, that can be used to act on the real world. Devices of this type are also called embedded systems due to the presence of the main operating unit, i.e. the built-in processor [6].

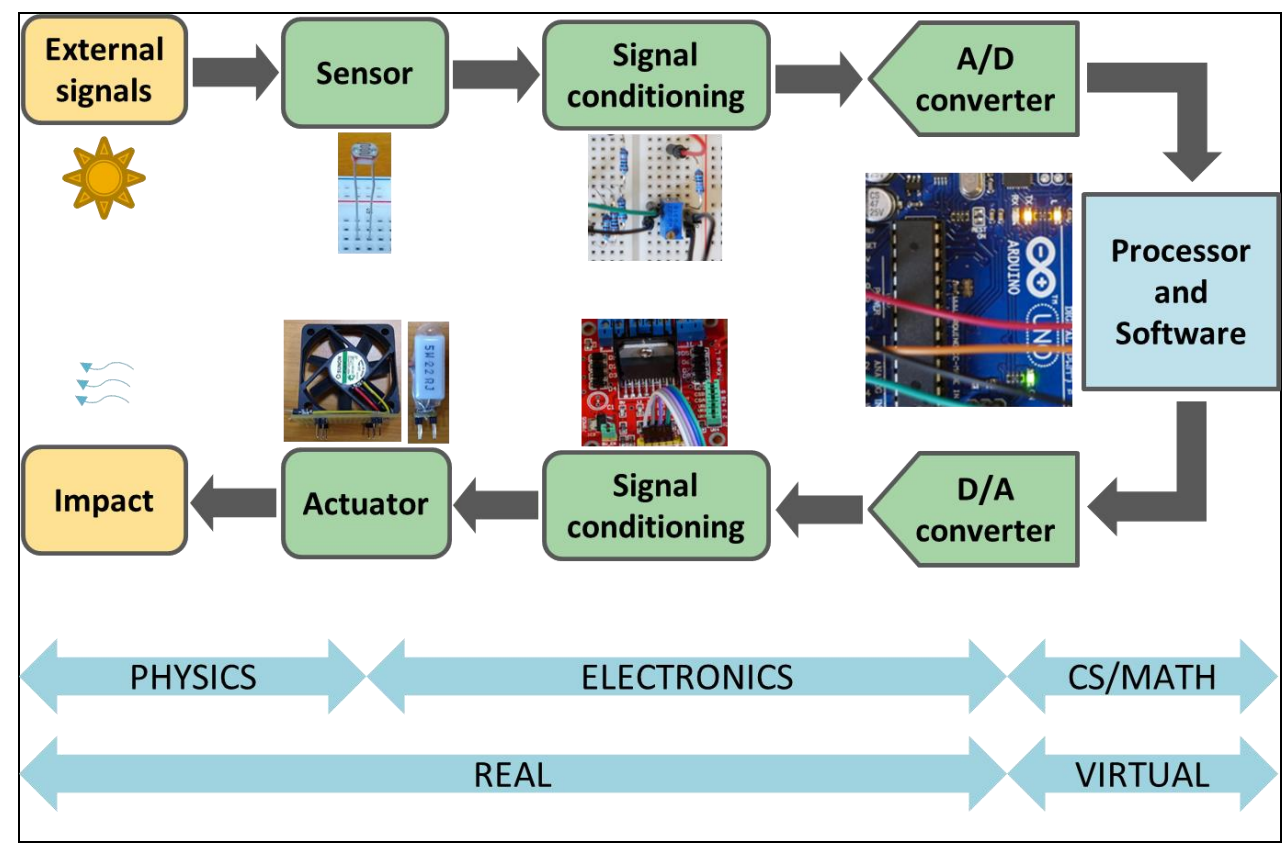

Figure 1. Block diagram of modern electronic devices.

This structure can be easily seen in many educational devices too, the teachers and students can make such systems using the available building blocks. They can design the algorithm of operation and write the corresponding code.

It is important to note that reliability is essential for all devices, embedded systems. Air bags must work in the right time, during patient monitoring software or hardware failure can risk life, but we also expect that mobile applications won't drive us in the wrong direction in a one-way street, that they make bank transfer to the right account and keep our data private. The catastrophe of a rocket is often mentioned as a typical example how a huge disaster can be caused by a simple inattention during the software code development [7]. In consequence, it is essential to develop a careful, 
technology-oriented approach and attitude that directly appears in the official requirements of education output. It is instructive to see what standards apply to IT teachers in our country. Here we highlight a few things that are more directly related to the above:

- Encourages students to form their own opinions, helps to develop critical thinking, with particular emphasis on drawing the attention to the dangers of IT applications.

- Has the knowledge to enable learning and interpreting the new results in the IT field. Knows the basic research methodology of the field.

- Able to find and integrate the knowledge of different fields, especially mathematics and natural sciences.

- Able to apply the theoretical knowledge acquired in his specialty in practice, to convey it to the students.

- Is aware of the fact that knowledge and competencies developed in the field affect other fields as well.

- Able to use the tools of informatics education of the school professionally, to use these in teaching and distance education. Able to develop curriculum for informatics and to support the application of IT in the development of other curricula.

- Works with teachers of related fields. Able to coordinate the scheduling of teaching the subjects also present in related subjects.

- Committed to demanding teaching and continuous self-education.

The standards of required professional knowledge is extensive and includes the technical fields, robotics too.

\subsection{Popular technical informatics education tools}

Increasingly better, cheaper and more efficient tools are available that supports the learning about the architecture and operating principles of today's devices in a playful, enjoyable and practical way. Lego robots [1]], single-board computers like, micro:bit [2], Arduino [3] and the Raspberry Pi [4] are good examples. They can handle many different sensors; several kinds of signals can be acquired. Students have direct access to the raw data what they can process, and they can produce impact as well accordingly. Therefore, the whole system is very transparent, the function of the sensors and other components can be clear even without knowing the details of the electronics background. Of course, there are many different levels of knowledge, but teachers must have indepth education, they should not be confused, if the student ask, for example, how a robot can sense the nearby objects. Teaching of the course called "Technical applications of informatics" for the informatics teacher students at our university takes 15 and 30 hours per semester concerning the basics of theoretical background and practice, respectively. The curriculum includes robotics, application of the Raspberry PI, and particularly based on the Arduino platform, which is extremely popular worldwide.

\subsection{Arduino applications - advantages and drawbacks}

Arduino is development platform based on a single-board computer hardware and a simple userfriendly software development environment. It is very transparent, practically it is a microcontroller (a single chip integrating a CPU and peripherals) whose pins are connected to easy-to-use pin headers. Many additional components, sensors, actuators are available, therefore students can meet more directly with electronics and circuits. In addition to this, the developers also solved the programming with a very good sense of proportion. The programming language is $\mathrm{C}++$, but in most

Central-European Journal of New Technologies in Research, Education and Practice

Volume 1, Number 1, 2019. 
cases, it is sufficient to know the most general parts common in other languages as well. The environment is kept simple, there are a limited number of functions, but they are really useful. The architecture of the programs fits well to the application principles of the microcontrollers without using operating systems (setup and loop functions replace the main function). The microcontroller is a professional component of commercial, industrial and medical technology, essential in embedded systems, were reliability, following the well-established principles, standards and rules are all of particular importance. Of course, all of these can't be expected, but careless, improper use and development of undesired attitude should be avoided in any case.

There are a huge number of solutions for almost any task and problem on the internet. A considerable part of these are developed by hobbyists, students, so not by experts. There are many smart ideas, but sometimes the drawbacks are substantial especially regarding high quality education. On one hand, it can motivate the students to reproduce instead of working out own version, in most cases they don't even understand why the teacher asks them to solve a problem if there are available solutions of the internet. On the other hand, due to the lack of comprehensive expertise of hobbyists, there is a large number of virtually working but technically incorrect solutions. The numerous hits for a single problem can make it very hard to find the right one even for an experienced teacher. Unfortunately, even the official Arduino pages and the textbooks show sometimes inadequate guidelines what can cause hard-to-change habits. Therefore, we consider it important to help understanding the operation and principles as much as possible, to show the professionally correct applications, to develop self-reliance and care. We do not aim to provide step-by-step instructions, rather we show cases studies as examples to demonstrate the application of the right methods and approach. During the laboratory practicing the use of embedded software and hardware development methods mean experimental learning, help to be confident when problems have to be solved. In accordance with the requirements of education output, cautious thinking and right attitude can be improved, including careful consideration of the problem, ability to find a right solution, proper application of the professional methods, testing, finding and fixing the problems efficiently.

\section{Arduino exercises}

Laboratory exercises for the informatics teacher students are related to several different fields. Students work with various sensors, circuits, mechanical parts and see several methods and approaches. They acquire fire and working safety knowledge, they learn to record the phases of the work and practice collaboration. The lecturer evaluates and scores their work. The students are free to select a solution for the given problem; they can use any of the supporting material available on the internet. The selected problems effectively develop their problem-solving skills. First, they can give a simpler solution, then they are asked to develop a more advanced and more professional version. In order to support this the lecturer can give some guidelines and explanations. It is important to note that they experience how to work in a laboratory environment equipped with instruments and other hardware. They learn that there are important rules and standards in order to guarantee the required quality and reliability even if the reason is not necessarily clear at first sight. In the course we have used the following exercise examples that demonstrate how solutions need some knowledge of mathematics, physics and occasionally some other disciplines, what can be somewhat surprising for the students.

Central-European Journal of New Technologies in Research, Education and Practice

Volume 1, Number 1, 2019. 


\subsection{Measurement of voltage and resistance}

Students certainly meet the problem of voltage measurement in most Arduino applications related to analogue signals. This is aided by the analogue-to-digital converter (ADC) of the microcontroller. Students learns the principle of converting a real value to integer number, they experience the effects of quantization and sampling, they understand the role of the voltage reference. The voltage can be expressed as []]:

$$
V_{\text {in }}=x \cdot \frac{V_{r e f}}{2^{b}},
$$

where $x$ is the integer code at the output of the ADC, $V_{\text {ref }}$ is the reference voltage (its default nominal value is $5 \mathrm{~V}$ for Arduinon Uno), $b$ is the number of bits of the output code (10 for Arduino Uno, so the voltage resolution is $\left.V_{r e f} / 1024\right)$. The task was to build a simple voltage divider circuit on a breadboard, whose output was connected to one of the analogue inputs of the Arduino board. The students had to write a code to sample and calculate the voltage using equation 1.

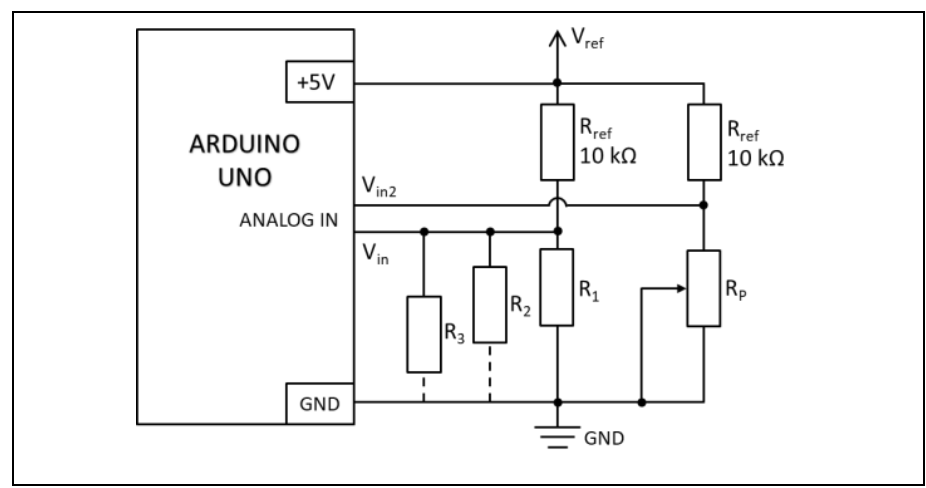

Figure 2. Voltage divider circuit connected to the Arduino board. One can select a resistor to measure any of the $R_{1}, R_{2}$ and $R_{3}$ by connect its lower end to the GND.

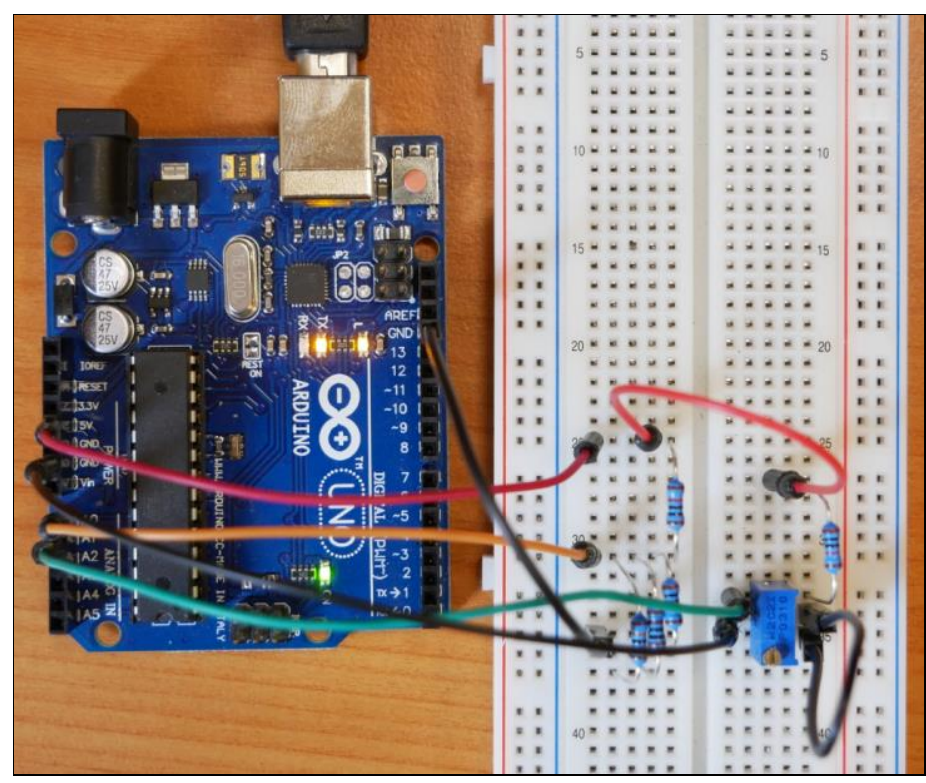

Figure 3. Voltage divider circuit assembled on a breadboard.

The Arduino integrated development environment (IDE) provides a so-called Serial monitor tool that can display the data sent by the microcontroller using only a few simple functions of the Serial library [2]. This is a quick method to see how the system is working and to find the possible errors. Central-European Journal of New Technologies in Research, Education and Practice 
The next task was to measure resistance. The voltmeter code had to be modified, but the students had to know and apply Ohm's law properly. The value of the resistor connected to ground (GND) can be obtained by measuring the voltage on it [10]. The same current flows through both resistors, its value can be easily obtained as $V_{r e f} V_{\text {in }} / R_{r e f}$. Finally, the unknown resistance is given as the voltage on it divided by the current:

$$
R=\frac{R_{r e f} \cdot V_{i n}}{V_{r e f}-V_{i n}},
$$

where $R_{r f}$ is a known reference resistor, $V_{r f}$ is the reference voltage, $V_{i n}$ is the measured voltage. During the exercise the students had to measure the value of three resistors and to display the value of the potentiometer in real time. They got help where to find the Arduino library functions [2] required to start a conversion and to read the ADC code and to send the data to the host computer over the serial port. Student were facing the problem of integer division, where undesired rounding occurred and caused large error in the measurement. They have solved the problem by using floating point constant for numbers that could be represented also be integers (e.g. $5 \mathrm{~V}$ reference voltage). Some time was needed to find the reason of this error, but the success was a good experience for them. Being informatics teacher students, they were a bit less familiar with the electronics part, but they understood the main principles after completing the exercise.

\subsection{Gate controlling system}

Exercises that model the operation of an ordinary system are particularly useful. This directly demonstrates practical usefulness and the underlying principles. A related task was to assemble a model of a garage door actuator system and to write the operating code.

Moving a stepper motor had to be realized by working out the appropriate algorithm based on the knowledge of the operation principle and driving methods. The rotation of the gate between 0 and 90 degrees were provided by the stepper motor. This is also a good example of the possibility of theoretical abstraction, since in reality not a stepper is used for the purpose, but it only means the management of a component, the main principles are not affected. It was also a part of the task to implement an emergency stop using an optical sensor. If an obstacle was detected, the movement had to be stopped. In order to make a photogate they got a photocell whose resistance had to be measured. They built the circuit and wrote the corresponding code. Opening of the gate was started by pressing a button connected to one of the pins of the Arduino board configured as digital input.

Central-European Journal of New Technologies in Research, Education and Practice

Volume 1, Number 1, 2019. 


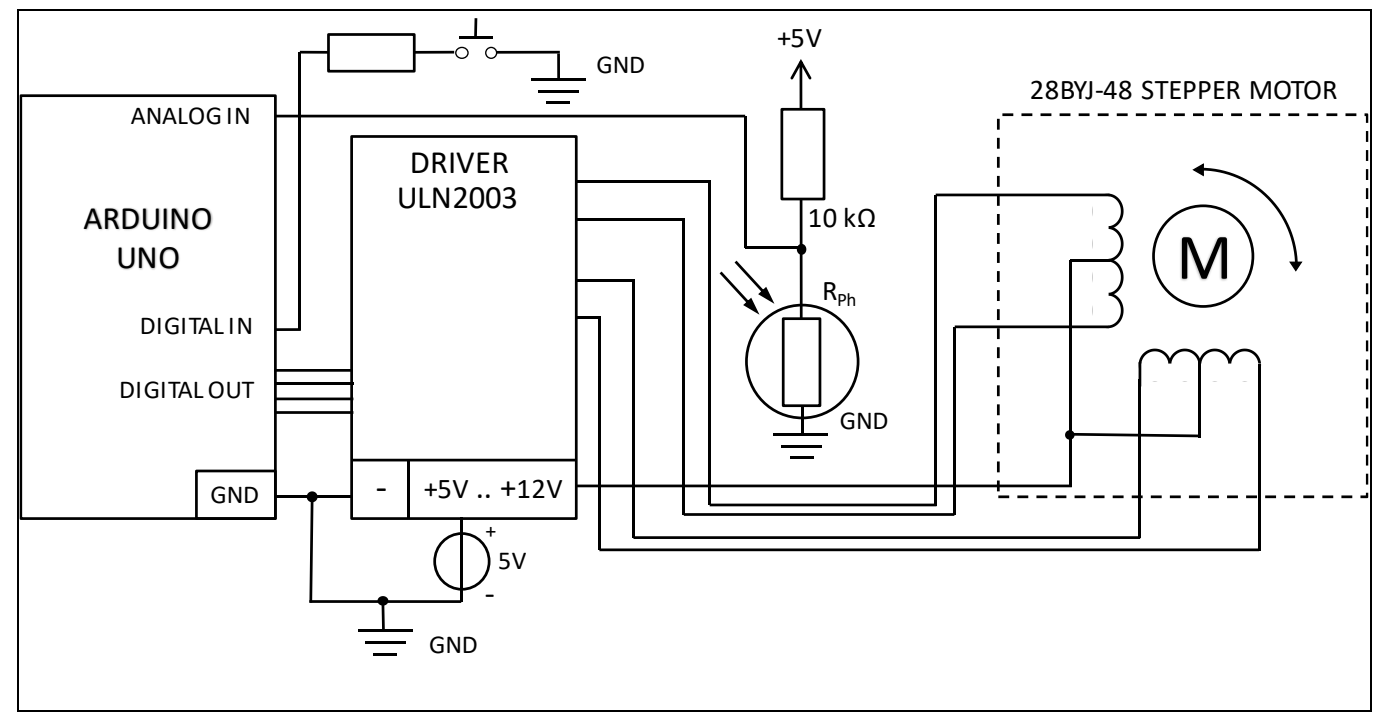

Figure 4. Arrangement of the gate controller system. The Arduino board controls a stepper motor using driver circuit. The gate opens upon pressing a button and the movement is suspended if the photogate detects an obstacle.

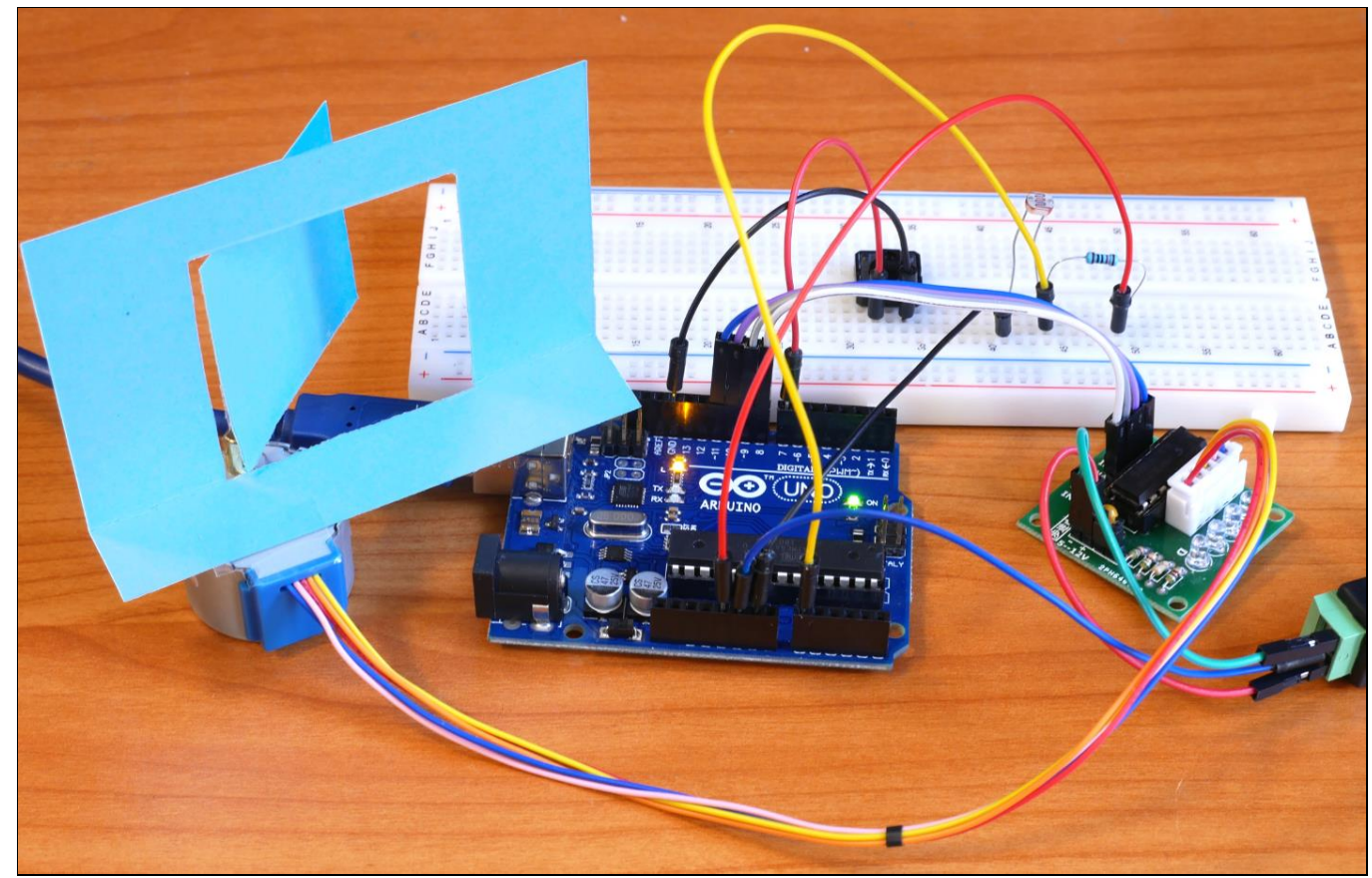

Figure 5. The realized gate controller model.

The task provides various options for solving the operation of the individual components. Half or full step can be used to control the motor, the speed of rotation can be changed, end positions can be detected. The photoresistor can be replaced by a phototransistor; infrared light can be used instead of visible light. The opening process can be controlled remotely; the operating logic can be varied. The movement speed can be reduced when approaching the end positions, a flashing LED can indicate that the gate is moving. The students usually write a for loop to do the required number of steps, however, the loop function can also be used with properly implemented conditional execution. Timing of the stepping can be solved more precisely using timer peripheral and interrupt techniques. These possibilities provide various levels of complexity to accommodate to students with different skills and experience. According to our observations the students had the following main difficulties: to understand the gearing of the stepper motor; to suspend the movement before 
starting and during the presence of an obstacle. The mechanical construction and driving principle of the stepper motor was new to the students. The microcontroller code runs without an operating system, which was also unusual. Some more tutorials and simulations [11] can aid the understanding of the operation principles of the stepper motors. Examples and more detailed explanation of the loop function can also be useful to help straightforward implementation of suspending the motion.

\subsection{Heart rate measurement using photoplethysmography}

Today's smartphones and smart watches are capable of heart rate measurements by using one of the suitable principles, the so-called photoplethysmography. An exciting exercise can be built up to measure heart activity by an Arduino board too.

The principle of the measurement is rather simple: direct an infrared light into the finger and measure the intensity of the reflected or passing through light. This depends on the instantaneous blood volume in the finger $[\underline{12}, \underline{13}]$. Therefore, one can get a time dependent signal mainly proportional to the blood pressure changes, so it can be used to detect the heart beats. The changing components of the signal is very small; it is superimposed on a large mean (DC) value. The students learn why and how to remove the large DC component and what are the simple methods to amplify the signal to fit into the input range of the ADC. The principle is rather general, it is applied to handle the signals of different sensors, e.g. a microphone. Building the circuit is a bit more what can be expected from the student during the lecture therefore in order to support the experimental work we provided our EDAQuino "shield" (plug-in board) that incorporates all of the required sensors and circuits $[\underline{14}, \underline{15}, \underline{16}]$. Note that if longer time is available, students can build the circuit on a breadboard, so they can learn and experience more about electronic signal processing. Details can be found in our recent related article [17].

The students had to implement periodic sampling of the time dependent signal. This can be implemented in a simple and common way, however one should know the limitations of the method [17]. The Arduino IDE provides a simple chart recorder feature with the so-called Serial plotter tool to display the signal in real time. This allows the students to have an early success by visualizing their own heart function and they will be motivated to continue with the more complicated tasks too. Real-time heartbeat detection can be implemented in various ways, and a LED can be toggled to indicate when a heartbeat occurs. The task can be extended considerably, if the heartbeat time instants are available. Several heart rate variability indicators used in the medical practice can be computed including the mean (mean RR) and standard deviation (sdRR) of the beat-to-beat intervals (RR intervals) and the proportion of the intervals longer than the previous one by more than $50 \mathrm{~ms}$ (pNN50) [18, 19, 19]. Histogram of the RR intervals can also be calculated and displayed.

Central-European Journal of New Technologies in Research, Education and Practice

Volume 1, Number 1, 2019. 


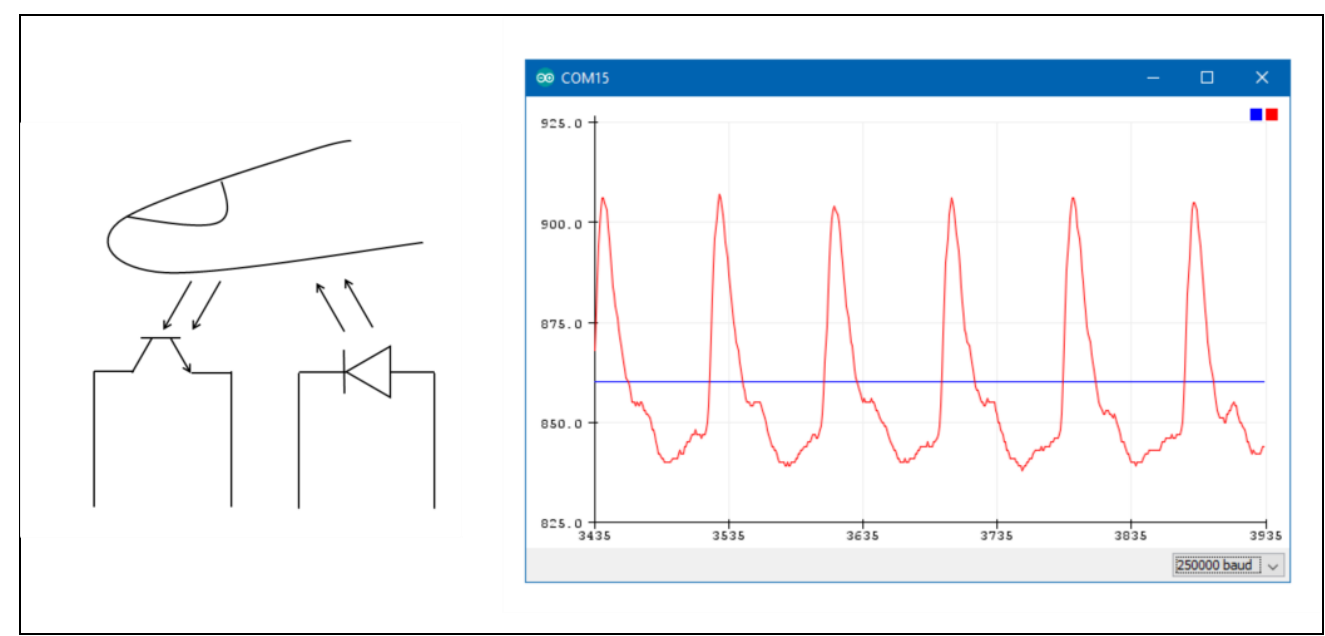

Figure 6. On the left-hand side, the arrangement of the finger photoplethysmography is shown. The plot on the right-hand side shows the Arduino's Serial plotter display of the phototransistors' output signal.

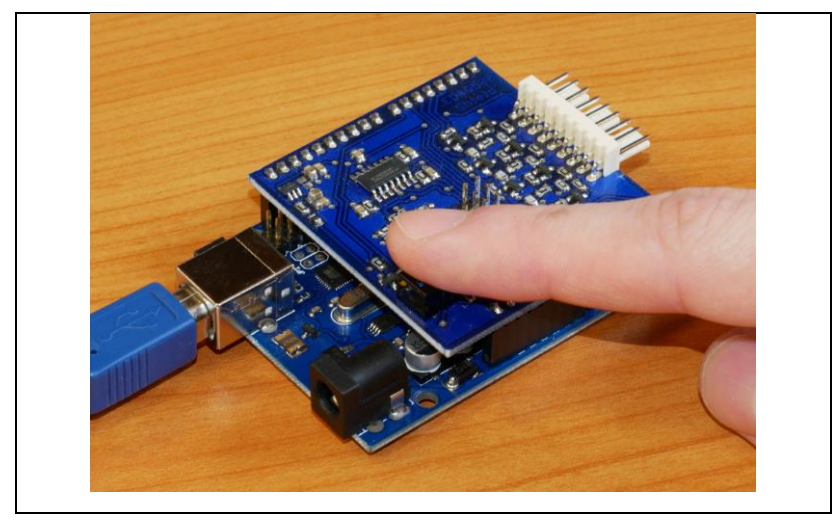

Figure 7. The students used the EDAQuino shield as a photoplethysmograph device.

We have found that designing and implementing the level crossing detection algorithm was not so easy for the students. This was mainly due to the lack of experience in real-time digital processing and the special programming requirements of the microcontroller were still not easy to follow. This highlights what could be the part of related education and what could be practiced during the teaching of different courses including introductory subjects of programming.

\subsection{Temperature regulation}

One of the simplest and most common method to keep something at the desired level is the onoff control applied in many everyday devices and systems. According to the general method of regulation, the deviation from the desired value has to be measured and increasing or decreasing should be forced accordingly. For on-off control, the strength of the effect is given, it does not dependent on the magnitude of the deviation from the desired value. In order to implement the on-off control, students used a power resistor that can be energized by applying voltage on it. The heating power depends on the voltage as follows:

$$
P=\frac{V^{2}}{R},
$$


where $V$ is the heating voltage, $R$ is the value of the power resistor that acts as a heating element.

This needs considerable current, that can be provided by external power circuits driven by some of the digital outputs of the Arduino board. A cooling can be solved by the use of a small fan or just by switching the heating off. A thermistor [21] (temperature dependent resistor) was used as temperature sensor. By measuring the resistance of this sensor, the temperature can be calculated using the following formula:

$$
T=\frac{1}{\frac{1}{T_{25}}+\frac{1}{B} \cdot \ln \left(\frac{R}{R_{25}}\right)},
$$

where $T$ is the temperature expressed in Kelvins, $T_{25}$ is the room temperature ( 25 degrees centigrade) in Kelvins $(298.15 \mathrm{~K}), B$ is a material constant of the thermistor, $R_{25}$ is the resistance of the thermistor at the $T_{25}$ room temperature. The resistance measurement was already discussed in Section 3.1. Following that the resistance of the thermistor can be expressed as:

$$
R_{t}=R_{\text {ref }} \frac{V}{V_{\text {ref }}-V}
$$

The task was to write a program that heats up the power resistor to a given temperature, and this temperature had to be kept. Students had to use two levels to switch the heating and cooling on and off, and they had to monitor the temperature by sending the data to the Serial plotter in real time. They could observe the effect of the levels on the accuracy of regulation. The code can be easily changed to implement pulse width modulation (PWM) driving that effectively allows different levels of impact using a two-state signal. The experiment can be realized in other systems including charging a capacitor to a certain level via a resistor. In this case only a capacitor and a resistor is required provided that the resistor value is high enough to limit the charging current to a safe level [22].

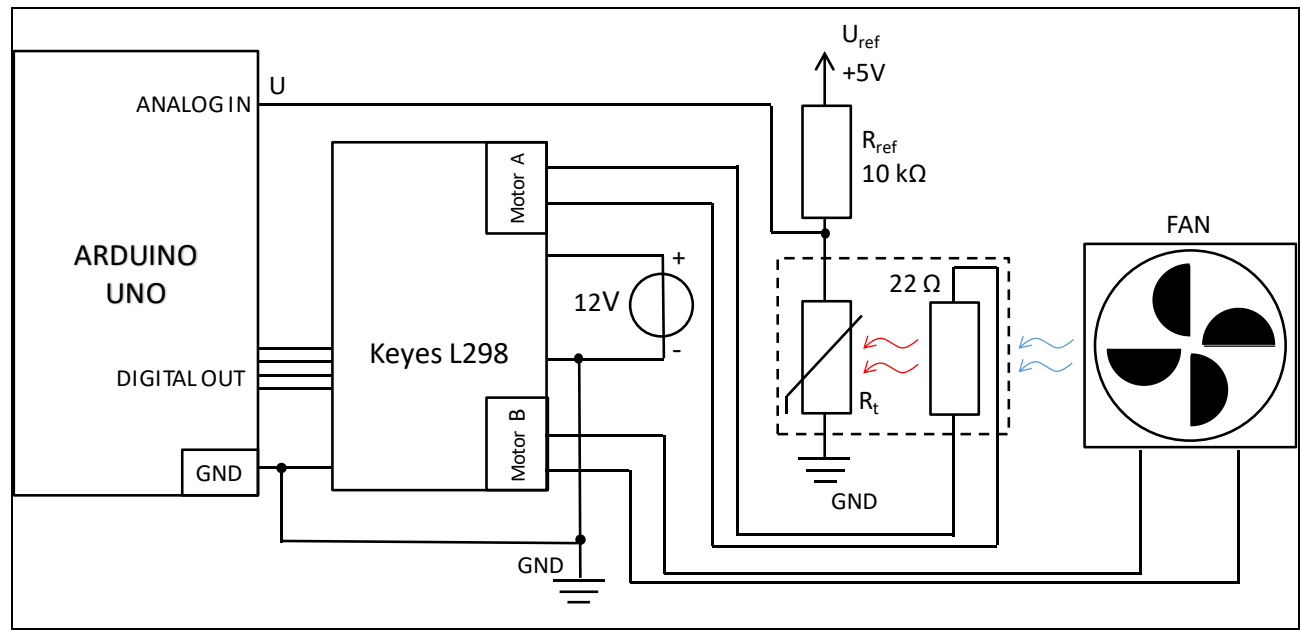

Figure 8. Block diagram of the system used to practicing on-off control. The Arduino board can switch on and off the heating and cooling via a higher power driver circuit (L298). 


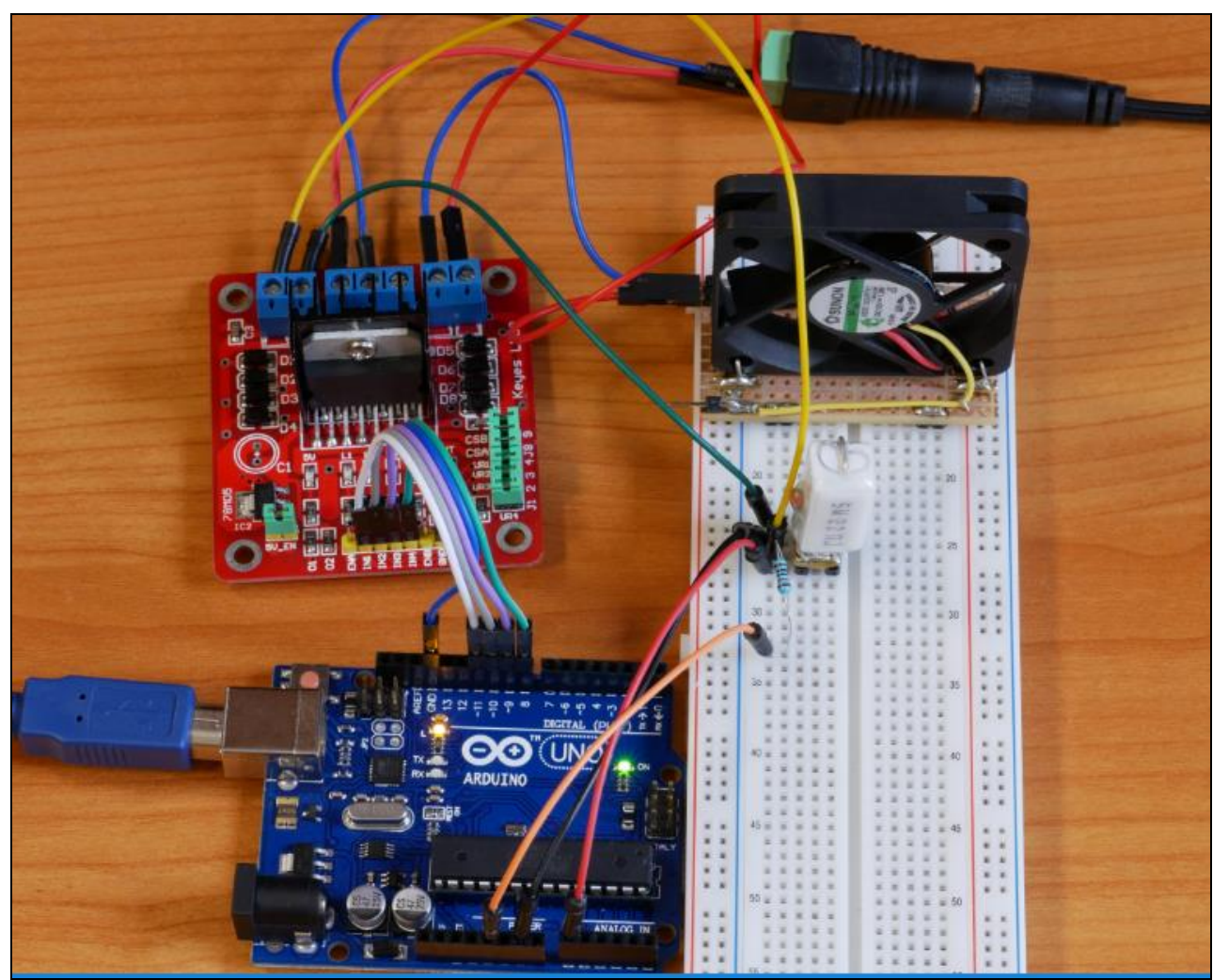

Figure 9. The assembled temperature control system.

We have experienced that one problem was to implement the hysteresis properly, and it was even harder to understand why the signal can be out of the levels for a short time and shown in Figure 10. Some everyday examples may help to understand the phenomenon better.

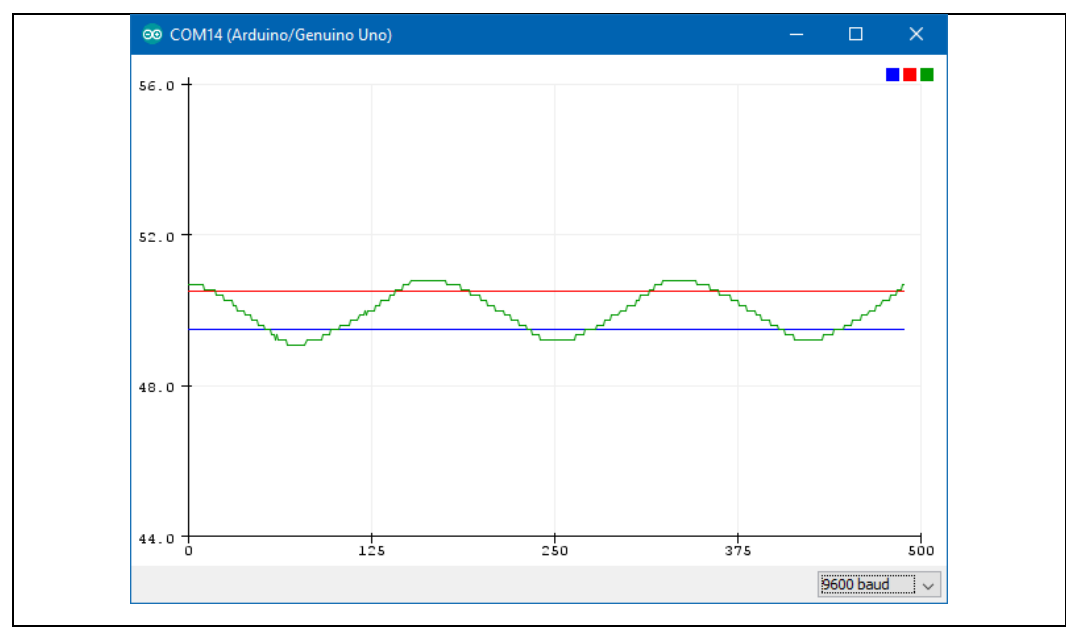

Figure 10. Temperature dependence during regulation (green curve). The levels of switching the heating and cooling on and off were $49.5^{\circ} \mathrm{C}$ and $50.5^{\circ} \mathrm{C}$

(blue and red lines).

During the implementation of Equation 4. sometimes integer division caused a problem, since in $\mathrm{C}$ and $\mathrm{C}++$ the result of dividing two integers is an integer too, and it can result large errors. Emphasis should be placed on teaching the most important principles of programming embedded microcontrollers to avoid introducing errors during coding [23]. Note also, that this was the first course for the students to meet simple electronics, making the circuits using breadboards. 


\section{Conclusion}

An important part of the training of informatics teacher students is to teach about the operation principles of the common electronic devices operated by processors and software. IT and other technical solutions are gaining importance in other areas too, so interdisciplinary nature is becoming widespread. Due to the rapid development of tools and software, it is essential to become more familiar with the universal principles and creative application of these. This can be effectively supported by laboratory practices in addition to theoretical education. Students can become more confident and demanding by gaining practical experience in a wider range, their problem-solving skills can be developed significantly.

We have shown four laboratory exercises for the Arduino platform used in the training of informatics teacher students. According to our experience, they could complete the tasks and found these interesting and exciting. The exercises can be expanded and used both in high school and university environments at various levels of complexity. Arduino circuits and accessories are readily available at a low cost, so classrooms can be equipped easily, a separate kit can be provided for every student. Students can use the same tools at home, which helps to be prepared and to realize their own ideas as well.

\section{Acknowledgments}

This study was funded by the Content Pedagogy Research Program of the Hungarian Academy of Sciences.

\section{References}

1. Information pages of Lego robots https://www.lego.com/en-us/mindstorms, (last viewed: 29.04.2019)

2. Information pages of the Micro:bit https://microbit.org/hu/, (last viewed: 29.04.2019)

3. Information pages of the Arduino https://www.arduino.cc/, (last viewed: 29.04.2019)

4. Information pages of the Raspberry $\mathrm{Pi}$ https://www.raspberrypi.org/, (last viewed: 29.04.2019)

5. Homepage of the HAS Research Group of Technical Informatics Methodology http://www.inf.u-szeged.hu/miszak/ (last viewed: 29.04.2019)

6. Embedded systems https://en.wikipedia.org/wiki/Embedded system, (last viewed: 29.04.2019)

7. Rocket disaster https://hownot2code.com/2016/09/02/a-space-error-370-million-for-an-integer-overflow/, (last viewed: 29.04.2019)

8. Zoltán Gingl, Gergely Makan, János Mellár, Arduino data conversion -1023 or 1024? , (2018) DOI:10.6084/m9.figshare.7434074.v1

9. Arduino functions https://www.arduino.cc/reference/en/, (last viewed: 29.04.2019) 
10. Gergely Makan, Róbert Mingesz, Zoltán Gingl, How accurate is an Arduino Ohmmeter?, (2019) Phys. Educ. 54 033001., DOI:10.1088/1361-6552/ab0910

11. Stepper motor

https://en.wikipedia.org/wiki/Stepper motor, (last viewed: 29.04.2019)

12. Nagy, Tamás, and Zoltán Gingl., Low-cost photoplethysmograph solutions using the Raspberry Pi, Computational Intelligence and Informatics, CINTI 2013, IEEE 14th International Symposium on. IEEE Budapest, Hungary (2013), pp. 163-167., DOI:10.1109/CINTI.2013.6705184

13. Zoltán Gingl, A photoplethysmograph experiment for microcontroller labs, INTERNATIONAL JOURNAL OF ELECTRICAL ENGINEERING EDUCATION (2012) 49 pp. 42-60., DOI:10.7227/IJEEE.49.1.4

14. Katalin Kopasz, Péter Makra and Zoltán Gingl, Edaq530: a transparent, open-end and opensource measurement solution in natural science education, Eur. J. Phys., (2011), 32 491, DOI:10.1088/0143-0807/32/2/020

15. Zoltán Gingl, János Mellár, Tamás Szépe, Gergely Makan, Róbert Mingesz, Gergely Vadai, Katalin Kopasz, Universal Arduino-based experimenting system to support teaching of natural sciences, GIREP-MPTL 2018 - Research and Innovation in Physics education:two sides of the same coin. 9th-13th July 2018, Donostia-San Sebastian, Spain (2018), DOI:10.6084/m9.figshare.6712541

16. Zoltan Gingl, Janos Mellar, Tamas Szepe, Gergely Makan, Robert Mingesz, Gergely Vadai, Katalin Kopasz, Universal Arduino-based experimenting system to support teaching of natural sciences, (2019) https://arxiv.org/abs/1901.03810; DOI: 10.1088/1742-6596/1287/1/012052

17. Zoltan Gingl, Gergely Makan, János Zsolt Mellár, Gergely Vadai, Robert Mingesz, Phonocardiography, photoplethysmography with simple Arduino setups to support interdisciplinary STEM education, (2018) DOI:10.6084/m9.figshare.7308356.v1

18. McKinley P S, Shapiro P A, Bagiella E, Myers M M, Meersman R E D, Grant I and Sloan $\mathrm{R}$ P, Deriving heart period variability from blood pressure waveforms, Journal of Applied Physiology (2003) 95 pp.1431-1438, DOI:10.1152/japplphysiol.01110.2002

19. Acharya U R, Joseph K P, Kannathal N, Lim C M and Suri J S, Heart rate variability: a review, Med Bio Eng Comput (2006) 44 pp.1031-1051, DOI:10.1007/s11517-006-0119-0

20. Tamás Nagy, Gergely Vadai and Zoltán Gingl, Digital phonocardiographic experiments and signal processing in multidisciplinary fields of university education, (2017) Eur. J. Phys. 38 055802, DOI:10.1088/1361-6404/aa7ae6

21. Thermistor https://en.wikipedia.org/wiki/Thermistor, (last viewed: 29.04.2019)

22. Zoltan Gingl, Robert Mingesz, Gergely Makan and Janos Mellar, Driving with an Arduino? Keep the lane!, (2019) Phys. Educ. 54 025010, DOI:10.1088/1361-6552/aafa41

23. Embedded C coding standards https://barrgroup.com/Embedded-Systems/Books/Embedded-C-Coding-Standard, (last viewed: 29.04 .2019$)$

\section{Authors}

\section{MAKAN Gergely}

University of Szeged, Department of Technical Informatics, Hungary, e-mail: makan@inf.u-szeged.hu

Central-European Journal of New Technologies in Research, Education and Practice

Volume 1, Number 1, 2019. 
ANTAL Dóra

University of Szeged, Department of Technical Informatics, Hungary,

e-mail: antal74dora@gmail.com

\section{MINGESZ Róbert}

University of Szeged, Department of Technical Informatics, Hungary,

e-mail: mingesz@inf.u-szeged.hu

\section{GINGL Zoltán}

University of Szeged, Department of Technical Informatics, Hungary,

e-mail: gingl@inf.u-szeged.hu

\section{MELLÁR János}

University of Szeged, Department of Technical Informatics, Hungary,

e-mail: mellar@inf.u-szeged.hu

\section{VADAI Gergely}

University of Szeged, Department of Technical Informatics, Hungary, e-mail: vadaig@inf.u-szeged.hu

\section{KOPASZ Katalin}

University of Szeged, Department of Optics and Quantum Electronics, Hungary, e-mail:kopaszka@titan.physx.u-szeged.hu

\section{License}

Copyright (C) MAKAN Gergely, ANTAL Dóra, MINGESZ Róbert, GINGL Zoltán, KOPASZ Katalin, MELLÁR János, VADAI Gergely. 2019.

Licensee CENTRAL-EUROPEAN JOURNAL OF NEW TECHNOLOGIES IN RESEARCH, EDUCATION AND PRACTICE, Hungary. This article is an open access article distributed under the terms and conditions of the Creative Commons Attribution (CC-BY) license.

http://creativecommons.org/licenses/by/4.0/
About this document

Published in:

CENTRAL-EUROPEAN JOURNAL OF NEW TECHNOLOGIES IN RESEARCH, EDUCATION AND PRACTICE

Volume 1, Number 1. 2019.

ISSN: 2676-9425 (online)

\section{DOI:}

10.36427/CEJNTREP.1.1.385 